\title{
ON THE CONJUGATE OF BOUNDED FUNCTIONS ${ }^{1}$
}

\author{
BY S. K. PICHORIDES
}

Communicated July 2, 1974

ABSTRACT. If $f$ is a real $2 \pi$-periodic function such that $|f| \leqslant$ $k<\pi / 2$ and $\tilde{f}$ its conjugate, then $\|\sinh (\tilde{f} / 2)\|_{2}<(\cos k)^{-1 / 2}\|f / 2\|_{2}$.

Let $f$ be a real $2 \pi$-periodic function and $\widetilde{f}$ its conjugate. If $|f| \leqslant 1$ and $0<k<\pi / 2$, then $[3, \mathrm{VII}, 2.11]$

$$
\frac{1}{2 \pi} \int \exp (k|\widetilde{f}|) \leqslant \frac{2}{\cos k} \text {. }
$$

It follows from (1) that if $m(y)=|\{x:|\widetilde{f}(x)|>y\}|$ is the distribution function of $\tilde{f}$, then

$$
m(y) \leqslant \text { Const } / \exp (k y)
$$

In the case where $f$ is the characteristic function of a measurable set $E \subset[-\pi, \pi]$, E. Stein and G. Weiss proved that [2, Lemma 5]

$$
m(y) \leqslant \text { Const } \sin (|E| / 2) / \sinh (y / 2) \text {. }
$$

Moreover they gave an exact formula for $m(y)$, which shows that $m(y)$ depends on $|E|$ only.

In [3] the proof of (1) is based on Cauchy's theorem for holomorphic functions. The same method can provide an alternative proof of the theorem of Stein and Weiss mentioned above [1, III, 1.10]. It seems that it has passed unnoticed that this method also yields an inequality similar to (3) for all bounded functions. It is obvious that such an inequality considerably improves (2) for large values of $y$. A convenient way to formulate this result is the following

THEOREM. If $f$ is a real $2 \pi$-periodic function such that $|f| \leqslant k<$ $\pi / 2$ and $\tilde{f}$ its conjugate, then

AMS (MOS) subject classifications (1970). Primary 42A40, 44A15.

${ }^{1}$ This work was done in part while the author was a visitor at the Department of Mathematics, Université de Paris-Sud, 91405 Orsay, France.

Copyright $\odot 1975$, American Mathematical Society 


$$
\|\sinh (\tilde{f} / 2)\|_{2} \leqslant(\cos k)^{-1 / 2}\|f / 2\|_{2} \text {. }
$$

Proof. Let $u+i v$ be the holomorphic function in the open unit disc associated with $f$. The real part $(\cosh v) \cos u$ of $\cos (u+i v)$ is harmonic. Observing that $v(0)=0$ and integrating over $|z|=r<1$ we have

$$
\frac{1}{2 \pi} \int(\cosh v) \cos u=\cos u(0)=\cos \left[\frac{1}{2 \pi} \int_{-\pi}^{\pi} f(x) d x\right] \text {. }
$$

Using the obvious inequality $\cos u \geqslant \cos k$, we obtain

$$
\begin{aligned}
\cos k\|\sinh (v / 2)\|_{2}^{2} & \leqslant \frac{1}{4 \pi} \int(\cosh v-1) \cos u \\
& =\left[\cos u(0)-\frac{1}{2 \pi} \int \cos u\right] / 2 \\
& =\frac{1}{2 \pi} \int \sin ^{2}(u / 2)-\sin ^{2}[u(0) / 2] .
\end{aligned}
$$

Letting $r \rightarrow 1$ we obtain

$$
\|\sinh (\widetilde{f} / 2)\|_{2}^{2} \leqslant \frac{1}{\cos k}\left\{\frac{1}{2 \pi} \int \sin ^{2} \frac{f}{2}-\sin ^{2}\left[\frac{1}{2 \pi} \int \frac{f}{2}\right]\right\},
$$

from which (4) follows immediately.

REMARK. It is obvious that for any function $f$ with constant absolute value $k$ (i.e. $f=k(2 g-1)$, where $g$ is the characteristic function of a measurable set), (5) reduces to equality.

\section{REFERENCES}

1. Y. Katznelson, An introduction to harmonic analysis, Wiley, New York, 1968. MR 40 \#1734.

2. E. Stein and G. Weiss, An extension of a theorem of Marcinkiewicz and some of its applications, J. Math. Mech. 8 (1959), 263-284. MR 21 \#5888.

3. A. Zygmund, Trigonometric series, 2nd ed., Cambridge Univ. Press, New York, 1968. MR 38 \#4882.

LIBRARY: NUCLEAR RESEARCH CENTER "DEMOCRITOS", AGHIA PARASKEVI ATTIKIS, GREECE

Current address: Université de Paris-Sud, Centre d'Orsay, 91405 Orsay, Mathématique, Bâtiment 425, France 